\title{
A Possible Solution to the Tritium Endpoint Problem
}

\author{
G. J. Stephenson, Jr.* \\ Department of Physics \& Astronomy, \\ University of New Mexico \\ Albuquerque, NM 87131, USA \\ and \\ T. Goldman ${ }^{\dagger}$ \\ Theoretical Division, MS B283 \\ Los Alamos National Laboratory \\ Los Alamos, NM 87545, USA
}

October 6, 2018

\begin{abstract}
Scalar or right-chiral interaction currents may be expected to produce a neutrino coupled to the electron which is different from, and perhaps even orthogonal to, that coupled to the electron by the standard model weak interaction. We show that, using reasonable parameter values for such additional interactions, it is possible to generate a spectrum which, if analyzed in the manner commonly employed by experimental groups, produces a negative neutrino mass-squared.
\end{abstract}

${ }^{*}$ E-mail: GJS@hepv1.phys.unm.edu

†E-mail: GOLDMAN@T5.LANL.GOV 


\section{Introduction}

Since the report of a positive result for the neutrino mass in Tritium beta decay by the ITEP group [1], there have been a number of measurements [2, 3, 4, 5, 6, 6, 8], all of which disagree with the ITEP result and all of which, when analyzed under the assumption of a single massive neutrino, give the result, generally viewed as unphysical, that the best fit value for $m_{\nu}^{2}$ is negative ${ }^{n}$. This has been interpreted as an excess of counts near the end point, which could be modelled as a "spike" at the end point [3, 5]. Furthermore, the Los Alamos group [3] reported that the best fit value was dependent on the distance from the end point taken as a cut off for including data in the fit. This corresponds to the dependence on the retarding potential used in an integral experiment as reported by Mainz [7, 8].

The basic idea of using the end point of a beta ray spectrum to look for the possiblity of a neutrino mass goes back to Fermi's original paper [10]. It was known by the late 40's [11] that the dependence of the spectrum near the end point on the neutrino mass is influenced by the Lorentz nature of the Weak Interaction. After the introduction of parity violation, this issue was revisited, for a single massive neutrino, by Enz [12].

It is now known that there are three Standard Model (SM) neutrinos with masses that are small compared with half the $Z^{0}$ mass, and that the neutrinos produced by the standard Weak Interaction in conjunction with the electron, the muon and the tau correspond to three orthogonal (at least very nearly orthogonal) combinations of these mass eigenstates. Therefore, analyses of the Tritium spectrum need to be revisited, allowing for three separate mass eigenstates and the possibility that other, weaker interactions may also play a role. In a separate paper we look at this problem in more generality [13]. For this Letter, we confine our remarks to a simple argument which shows that it is possible to generate a spectrum which would produce the "negative mass-squared" effect that has been reported.

\section{The Effect of non-Standard Model Physics}

Interactions beyond the SM must be weaker, at low energies, than the usual left-chiral $\mathrm{SU}(2)$ to avoid serious conflict with existing data. Presumably this is due to the boson mediating the interaction being much heavier than the known W's and Z, and/or the coupling constant being smaller than that for the SM. Absent a particular Grand Unified Theory in which one wishes to embed the SM and the new interactions, that is all one can say.

For low energy physics, like nuclear $\beta$-decay in general and Tritium $\beta$-decay in particular, such new interactions can only appear as effective currents in the four fermion formulation of the theory with the usual space time structure of $S, P, T, V$ or $A$. Given the dominance of the SM, it is reasonable to recast this as $S, P, T, R$ or $L$, where $R=(V+A)$ and $L=(V-A)$.

\footnotetext{
${ }^{1}$ For a discussion based on a tachyonic interpretation, see [9].
} 
The effective low energy interaction Hamiltonian is

$$
H_{I}=\sum_{\alpha=S, P, R, L, T} G^{\alpha}\left(J_{h \alpha}^{\dagger} \cdot J_{l \alpha}+\text { h.c. }\right)
$$

where, for example,

$$
J_{l \alpha}=\overline{\psi_{l}} \Gamma_{\alpha} \psi_{\nu}
$$

with $\psi_{l}$ representing a charged lepton, $\psi_{\nu}$ a neutral lepton (see the discussion below for more detail), and a similar construction on the hadron side. Explicitly,

$$
\begin{aligned}
\Gamma_{S} & =1 \\
\Gamma_{P} & =\gamma^{5} \\
\Gamma_{R} & =\gamma^{\mu}\left(1+\gamma^{5}\right) / 2 \\
\Gamma_{L} & =\gamma^{\mu}\left(1-\gamma^{5}\right) / 2 \\
\Gamma_{T} & =\left[\gamma^{\mu}, \gamma^{\nu}\right] .
\end{aligned}
$$

For nuclear beta decay in the SM, only $\alpha=L$ survives and $G^{L}$ is related to the basic parameters of the SM by [14]

$$
G^{L}=V_{u d} \frac{\pi \alpha_{W}}{\sqrt{2} M_{W}^{2}}
$$

where $V_{u d}$ is the appropriate element of the hadronic CKM matrix [15], $\alpha_{W}$ is the fine structure constant for the $S U(2)_{W}$ of the SM, and $M_{W}$ is the mass of the usual $W^{ \pm}$.

If there are additional bosons that couple to the left-chiral fermion current, they can only renormalize $L$ and cannot lead to any new structure near the end point. For Tritium $\beta$-decay, the energy available for the kinetic energy of the resulting particles is approximately $18.65 \mathrm{keV}$. Conservation of momentum and energy limits the recoil energy of the molecular ion to a few electron volts. We may therefore ignore $P$ and $T$ and concentrate on the interference between $L$ and $R$ or $S$.

In this analysis we assume three mass eigenstates $\nu_{i}$ with eigenvalues $m_{i}$. The charge changing current of the SM produces, in conjuction with an electron, a linear combination of these mass eigenstates which we call an electron anti-neutrinot,

$$
\overline{\nu_{e}}=\sum_{i=1}^{3} \cos \theta_{i} \overline{\nu_{i}}
$$

where the $\cos \theta_{i}$ are the direction cosines with respect to the three orthogonal mass eigenstates. With this definition of $\overline{\nu_{e}}$, the expected form of the differential spectrum becomes

$$
\frac{d N}{d E_{\beta}}=K F\left(Z_{d}, E_{\beta}\right) q_{\beta} E_{\beta} E_{\nu} \sum_{i=1}^{3} \cos ^{2} \theta_{i} \sqrt{E_{\nu}^{2}-m_{i}^{2}} \Theta\left(E_{\nu}-m_{i}\right)
$$

\footnotetext{
${ }^{2}$ Whether the mass eigenstates are Dirac or Majorana particles, this expansion is valid for both neutrinos and anti-neutrinos. Where there is no likelihood of confusion, we refer to both as neutrinos.
} 
where $q_{\beta}$ is the electron momentum and we have explicitly written

$$
q_{\nu_{i}}=\sqrt{E_{\nu}^{2}-m_{i}^{2}}
$$

The $\Theta$ functions guarantee that each mass eigenstate contributes only above its threshhold in $E_{\nu}=E_{0}-E_{\beta}$, where $E_{0}$ is the end point energy appropriate to a massless neutrino, and $K$ is a constant incorporating the SM Weak Interaction coupling constant and the nuclear matrix elements. $F\left(Z_{d}, E_{\beta}\right)$ is the usual Fermi function incorporating the electromagnetic interaction between the outgoing $\beta$ and the daughter product denoted by $Z_{d}$.

Now consider the effect of another, weaker charge changing interaction, either $R$ or $S$. This will, in general, couple the electron to a different linear combination of neutrino mass eigenstates

$$
\tilde{\nu}_{e}=\sum_{i=1}^{3} \cos \tilde{\theta}_{i} \nu_{i}
$$

where the $\cos \tilde{\theta}_{i}$ are the direction cosines for this new vector.

One expects that interference effects between the new interaction and the SM, for reactions involving charged currents and an electron, are supressed by the overlap between $\nu_{e}$ and $\tilde{\nu_{e}}$ or by a mass weighted overlap [13]. However, that assumes that all mass eigenstates can participate. For most measurements, $E_{\nu} \gg m_{i}$ and this assumption is well founded. For $\beta$-decay end point studies, this assumption is invalid.

The form of Eq.(6) rests on the fact that it is the mass eigenstates that propagate away from the interaction. Therefore, the discussion of the effect on the spectrum, including interference between $L$ from the SM and the new interaction, involves a separate calculation for each mass eigenstate. In [13] we discuss the implications of this more fully, including the dependence on $E_{\beta}$. In this Letter we concentrate on the behavior within a small range below the end point (a few hundred eV) where the variations in $E_{\beta}$ are negligible. Given these assumptions, Eq.(6) may be generalized as

$$
\begin{aligned}
\frac{d N}{d E_{\beta}}= & K F\left(Z_{d}, E_{\beta}\right) q_{\beta} E_{\beta} E_{\nu} \times \\
& \sum_{i=1}^{3} \cos ^{2} \theta_{i}\left(1+\epsilon_{i}\right)\left(1+\frac{\phi_{i}}{E_{\nu}}\right) \sqrt{E_{\nu}^{2}-m_{i}^{2}} \Theta\left(E_{\nu}-m_{i}\right)
\end{aligned}
$$

where $\epsilon_{i}$ and $\phi_{i}$ depend on the Lorentz structure of the new interaction. Specifically, if

$$
\eta=\left(\tilde{\alpha} / \alpha_{W}\right)\left(M_{W}^{2} / \tilde{M}^{2}\right)\left(\cos \tilde{\theta_{d}} / \cos \theta_{d}\right)
$$

where, as before, $\alpha_{W}$ is the usual Weak Interaction fine structure constant [14], $\tilde{\alpha}$ that for the new interaction, $M_{W}$ is the mass of the usual $W^{ \pm}$bosons and $\tilde{M}$ is the mass of the charged bosons mediating the new interaction. $\cos \theta_{d}$ and $\cos \tilde{\theta}_{d}$ are the analogous direction cosines in the hadronic sector 1 .

\footnotetext{
${ }^{3}$ In the hadron sector, these direction cosines are usually expressed, for the SM, as elements of the CKM matrix 15 .
} 
Then, for $R$,

$$
\begin{aligned}
\xi_{i} & =\eta\left(\cos \tilde{\theta}_{i} / \cos \theta_{i}\right) \\
\phi_{i} & =\frac{2 m_{i} \xi_{i}}{\left(1+\epsilon_{i}\right)}\left(\frac{m_{e}}{E_{0}}\right) \\
\epsilon_{i} & =\xi_{i}^{2}
\end{aligned}
$$

while for $S$,

$$
\begin{aligned}
\xi_{i} & =\eta\left(\cos \tilde{\theta}_{i} / \cos \theta_{i}\right) \\
\phi_{i} & =-\frac{m_{i}\left(\xi_{i}+\frac{2 \xi_{i}^{2} m_{e}}{E_{0}}\right)}{1+\epsilon_{i}} \\
\epsilon_{i} & =\xi_{i} \frac{m_{e}}{E_{0}}+2 \xi_{i}^{2}
\end{aligned}
$$

The sign in Eq.(12) comes from performing the usual traces, but the overall sign of the $\xi_{i}$ is indeterminate due to the various direction cosines. (There is, in fact, additional freedom due to purely left- or right-chiral couplings of $\mathrm{S}$ to leptons[16], which are treated in [13].)

\section{$3 \quad$ Effect on Differential Spectra}

Let us now assume, for simplicity, that only one mass eigenstate is important for the fit near the end point, given the resolution of current experiments. Let that mass eigenvalue be denoted as $m_{1}$, and scale $E_{\nu}$ and the $\phi_{i}$ in units of $m_{1}$,

$$
\begin{aligned}
x & =E_{\nu} / m_{1} \\
f_{i} & =\phi_{i} / m_{1}
\end{aligned}
$$

In this case, Eq.(9) becomes

$$
\frac{d N}{d E_{\beta}}=K^{\prime}\left(1+\epsilon_{1}\right) x^{2} \cos ^{2} \theta_{1}\left(1+\frac{f_{1}}{x}\right) \sqrt{1-\frac{1}{x^{2}}} \Theta(x-1)
$$

If we were to fit this with a formula for the spectrum which is derived under the assumption that there is only one neutrino and that it has a mass extracted from the spectrum as $<m^{2}>_{\text {fit }}$, we would fit Eq.(14) with the function

$$
\frac{d N}{d E_{\beta}}=K^{\prime}\left(1+\epsilon_{1}\right) x^{2} \sqrt{1-\frac{<r^{2}>_{f i t}}{x^{2}}}
$$

where $<m^{2}>_{f i t}=m_{1}^{2}<r^{2}>_{f i t}$ is the extracted (apparent) value of the neutrino mass-squared. 
Setting Eqs (14) and (15) equal, at some particular value of x, gives an equation for $<r^{2}>_{f i t}$,

$$
\begin{aligned}
<r^{2}>_{f i t}= & x^{2}\left[1-\cos ^{4} \theta_{1}\right] \\
& -x\left[2 f_{1} \cos ^{4} \theta_{1}\right] \\
& +\left(1-f_{1}^{2}\right) \cos ^{4} \theta_{1} \\
& +x^{-1} 2 f_{1} \cos ^{4} \theta_{1} \\
& +x^{-2} f_{1}^{2} \cos ^{4} \theta_{1}
\end{aligned}
$$

At $x=1$, precisely at the end point of the physical spectrum, Eq. (16) gives the result $<r^{2}>_{f i t}=1$. If the quantity $\cos ^{4} \theta_{1}$ is small compared to $1,<r^{2}>_{\text {fit }}$ will grow as $x$ increases. On the other hand, if this quantity is nearly 1 , which would occur if $\overline{\nu_{e}}$ is nearly a mass eigenstate, then the second term in Eq.(16) can dominate, leading to $<r^{2}>_{f i t}<0$ for some values of $x$. The value of $x$ at which $<r^{2}>_{f i t}$ again becomes positive is a sensitive function of $f_{1}$ and $\cos ^{2} \theta_{1}$.

\section{Effect on Integral Spectra}

To obtain sufficient statistics, experiments that measure differential spectra make a global fit to data over some range from the end point up to some value of $E_{\nu}$, which translates, in practice, to fitting over a range of $E_{\beta}$ down to some value. This is done automatically in those experiments that measure an integral spectrum.

In these cases, the fitting procedure, viewed in terms of theoretical constructs only and ignoring essential experimental details, corresponds to finding the value of $<r^{2}>_{\text {fit }}$ that minimizes the integral

$$
I=\int_{1}^{x^{c}} d x x^{4}\left(1+\epsilon_{1}\right)^{2}\left[\cos ^{2} \theta_{1}\left(1+\frac{f_{1}}{x}\right) \sqrt{1-\frac{1}{x^{2}}}-\sqrt{1-\frac{<r^{2}>_{f i t}}{x^{2}}}\right]^{2}
$$

\section{A Numerical Example}

To illustrate the effects on the extraction of $<m^{2}>_{f i t}$, we have chosen a particular example. This choice is motivated solely by pedagogy. We assume

$$
\begin{aligned}
f_{1} & =0.2 /(1.01) \\
\tilde{\theta_{1}} & =-\theta_{1} \\
\cos ^{2} \theta_{1} & =0.995
\end{aligned}
$$

The sign of $\tilde{\theta}_{1}$ plays no role here but, as shown in [13], when coupled with the assumptions that only two mass eigenstates contribute and $m_{2}=200 m_{1}$, it guarantees that the interference cancels far from the end point, leaving only a small normalization correction to the standard spectrum.

The actual effect on the spectrum is quite small. Nonetheless, the impact on $<m^{2}>_{\text {fit }}$ is marked, as can be seen in the Figure. To obtain that curve, we integrated 
the expression in Eq.( 17) to $x^{c}$ for a grid of $<r^{2}>_{f i t}$, then found, by a three point interpolation, the value that minimized $I$. For this example, one can clearly see the dependence of the extracted $<m^{2}>_{f i t}$ on the cut-off energy. Furthermore, if one argued for choosing a cut-off where the extracted value was most stable, one would obtain $<m^{2}>_{f i t} \approx-2 m_{1}^{2}$. In spite of the integration in Eq.(17), the sensitivity to parameter values discussed after Eq.(16) persists. This suggests the possibility of extracting $f_{1}$ and $\cos ^{2} \theta_{1}$ directly from a curve of $<m^{2}>_{\text {fit }}$ versus the cut-off energy.

\section{Conclusion}

In summary, any charge-changing interaction beyond the SM is likely to couple the electron to a different combination of neutrino mass eigenstates than that coupled to the electron by the SM. This can have the effect of decreasing expected interference signals in regimes where all mass eigenstates participate fully. Near the end point of a beta ray spectrum, however, some mass eigenstates may be kinematically excluded, restoring the visibility of interference signals.

We have recast the usual analysis in terms of the mass eigenstates to make this possibility more transparent, and have shown that it is quite possible to obtain values

of $<m^{2}>_{f i t}<0$. We therefore recommend that the form of Eq.(14) be used in the analysis of the Tritium $\beta$-decay spectrum.

\section{References}

[1] V. A. Lubimov et al. , Phys. Lett. 94B, 266 (1980); S. Boris et al. , Phys. Rev. Lett. 58, 2019 (1987); S. D. Boris et.al.,Pis'ma Zh. Eskp. Teor. Fiz. 45, 267 (1987) [JETP Lett. 45, 333 (1987)].

[2] M. Fritschi et al. , Phys. Lett. B173, 485 (1986).

[3] R. G. H. Robertson et al. , Phys. Rev. Lett. 67, 957 (1991).

[4] Wolfgang Stoeffl and Daniel J. Decman, Phys. Rev. Lett. 75, 3237 (1995).

[5] A. I. Belesev et. al., Phys. Lett. B350, 263 (1995).

[6] V. M. Lobashev it et al. , Neutrinos '96, Karl Enqvist, Katri Huitu and Jukra Maalampi, eds. (World Scientific, Singapore, 1997), p. 264.

[7] Ch. Weinheimer it et al. , Phys. Lett. B300, 210 (1993).

[8] H. Backe et al. , Neutrinos '96, Karl Enqvist, Katri Huitu and Jukra Maalampi, eds. (World Scientific, Singapore, 1997), p. 259.

[9] A. Chodos and A. Kostelecky, Phys. Lett. B336, 295 (1994); hep-ph/9409404.

[10] E. Fermi, Zeit. Phys. 88, 161 (1934); Nuovo Cim. 2, 1 (1934). 
[11] O. Koefed-Hansen, Phys. Rev. 71, 451 (1947); Phys. Rev. 74, 1785 (1948); Physica XVIII, 1287 (1952).

[12] C. P. Enz, Nuovo Cim. 6, 250 (1957).

[13] G. J. Stephenson Jr. and T. Goldman, in preparation.

[14] Howard Georgi, Weak Interactions and Modern Particle Theory, (Benjamin/Cummings, Menlo Park, 1984); T. Goldman, Adv. Nucl. Phys. 18, 315 (1987).

[15] N. Cabibbo, Phys. Rev. Lett. 10, 531 (1963); M. Kobayashi and K. Maskawa, Progr. Theor. Phys. 49, 652 (1973); L. Maiani, Phys. Lett. 62B, 183 (1976).

[16] Peter Herczeg, private communication.

\section{Figure Caption}

Figure 1. The value of $\left\langle r^{2}>_{f i t}\right.$ that minimizes the integral expression of Eq.(17) as a function of the cut-off energy expressed as $E_{\nu}^{c} / m_{1}$. The parameters of the calculated spectrum are given in the text; the value of $f_{1}$ has been rounded in the label. 


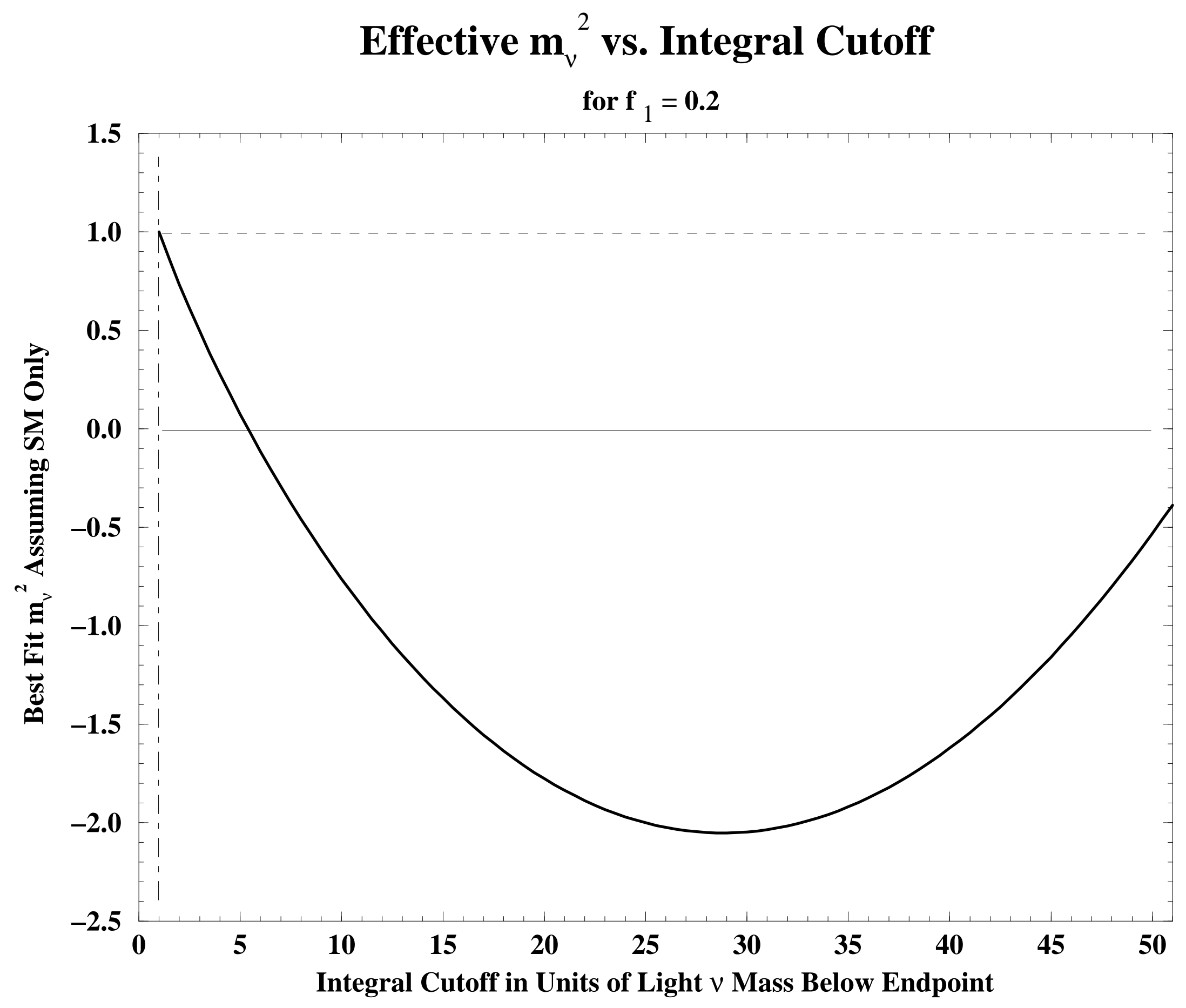

